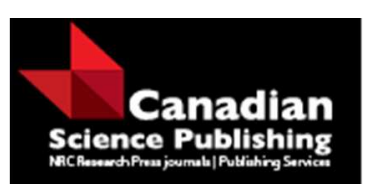

Canadian Journal of Physics

Revue canadienne de physique

\begin{abstract}
Asymptotic Safety in Quantum Gravity and Diffeomorphic Non-isometric Metric Solutions to the Schwarzschild Metric
\end{abstract}

\begin{tabular}{|r|l|}
\hline Journal: & Canadian Journal of Physics \\
\hline Manuscript ID & cjp-2017-0226.R1 \\
\hline Manuscript Type: & Article \\
\hline Date Submitted by the Author: & 27-May-2017 \\
\hline Complete List of Authors: & $\begin{array}{l}\text { Castro, Carlos; Clark Atlanta University, Center for Theoretical Studies of } \\
\text { Physical Systems }\end{array}$ \\
\hline $\begin{array}{r}\text { Is the invited manuscript for } \\
\text { consideration in a Special } \\
\text { Issue? : }\end{array}$ & $\begin{array}{l}\text { General Relativity, Black Holes, Asymptotic Safety;, Quantum Gravity, } \\
\text { Phase Space }\end{array}$ \\
\hline
\end{tabular}

SCHOLARONE"

Manuscripts 


\title{
Asymptotic Safety in Quantum Gravity and Diffeomorphic Non-isometric Metric Solutions to the Schwarzschild Metric
}

\author{
Carlos Castro Perelman \\ January 2017, Revised May 2017 \\ Center for Theoretical Studies of Physical Systems, Clark Atlanta University, Atlanta, \\ GA. 30314. perelmanc@hotmail.com
}

\begin{abstract}
We revisit the construction of diffeomorphic but not isometric metric solutions to the Schwarzschild metric. These solutions require the introduction of non-trivial areal-radial functions and are characterized by the key property that the radial horizon's location is displaced continuously towards the singularity $(r=0)$. In the limiting case scenario the location of the singularity and horizon merges and any infalling observer hits a null singularity at the very moment he/she crosses the horizon. This fact may have important consequences for the resolution of the fire wall problem and the complementarity controversy in black holes. This construction allows to borrow the results over the past two decades pertaining the study of the Renormalization Group (RG) improvement of Einstein's equations which was based on the possibility that Quantum Einstein Gravity might be non-perturbatively renormalizable and asymptotically safe due to the presence of interacting (non-Gaussian) ultraviolet fixed points. The particular areal-radial function that eliminates the interior of a black hole, and furnishes a truly static metric solution everywhere, is used to establish the desired energy-scale relation $k=k(r)$ which is obtained from the $k$ (energy) dependent modifications to the running Newtonian coupling $G(k)$, cosmological constant $\Lambda(k)$ and spacetime metric $g_{i j,(k)}(x)$. (Anti) de Sitter-Schwarzschild metrics are also explored as examples. We conclude with a discussion of the role that Asymptotic Safety might have in the geometry of phase spaces (cotangent bundles of spacetime); i.e. namely, in establishing a quantum spacetime geometry/classical phase geometry correspondence $g_{i j,(k)}(x) \leftrightarrow g_{i j}(x, E)$.
\end{abstract}

Keywords : General Relativity; Black Holes; Asymptotic Safety; Quantum Gravity; Phase Space. PACS : 04.60.-m, 04.65.+e, 11.15.-q, 11.30.Ly 


\section{Introduction : Diffeomorphic but Not Isometric Solutions to the Schwarzschild Metric}

In this introduction we review the key points in [3] which are essential to follow the next sections. The static spherically symmetric (SSS) vacuum solution of Einstein's field equations [1] that we learned from the text books is actually the Hilbert form of the original Schwarzschild [2] solution

$$
(d s)^{2}=\left(1-\frac{2 G M}{r}\right)(d t)^{2}-\left(1-\frac{2 G M}{r}\right)^{-1}(d r)^{2}-r^{2}(d \Omega)^{2} .
$$

There are an infinite number of metrics which are diffeomorphic but not isometric to the Hilbert form of the Schwarzschild [2] solution. A particular class of metrics are given by a family of metrics parametrized by a family of areal radial functions $\rho_{\lambda}(r)$ (in $c=1$ units), in terms of a real parameter $0 \leq \lambda<1$, as follows

$$
(d s)_{(\lambda)}^{2}=\left(1-\frac{2 G M}{\rho_{\lambda}(r)}\right)(d t)^{2}-\left(1-\frac{2 G M}{\rho_{\lambda}(r)}\right)^{-1}\left(d \rho_{\lambda}\right)^{2}-\rho_{\lambda}^{2}(r)(d \Omega)^{2} .
$$

where $\left(d \rho_{\lambda}\right)^{2}=\left(d \rho_{\lambda}(r) / d r\right)^{2}(d r)^{2}$ and the solid angle infinitesimal element is $(d \Omega)^{2}=$ $(d \phi)^{2}+\sin ^{2}(\phi)(d \theta)^{2}$. The surface area at each point $r$ is now given by $4 \pi\left(\rho_{\lambda}(r)\right)^{2}$ so that $\rho_{\lambda}(r)$ plays the role of an effective radius and hence the name of "areal-radial" function for $\rho_{\lambda}(r)$.

It is clear that the metric (1.2) is diffeomorphic but not isometric to the Hilbert form (1.1) of the Schwarzschild [2] solution because the area elements $r^{2}(d \Omega)^{2} \neq \rho_{\lambda}^{2}(r)(d \Omega)^{2}$ are not equal, except in the trivial case when $\rho_{\lambda}(r)=r$. The diffeomorphisms are simply established by the mappings $r \rightarrow \rho_{\lambda}(r)$. Therefore, despite that the metric (1.2) has the same exact appearance as the Schwarzschild solution, it is very different. We have not relabeled the radial variable $r$ by giving it another "name" and calling it " $\rho_{\lambda}$ ", because $\rho_{\lambda}(r)$ is itself a function of $r$. Furthermore, one has not performed a naive change of variables by writing $r=r\left(r^{\prime}\right)$ because $\rho(r) \neq r=r\left(r^{\prime}\right)$. The metric (1.2) leads to modifications of the Newtonian potential at short distances. One recovers the Newtonian potential in the asymptotic regime when $\rho_{\lambda}(r) \simeq r$.

In the Appendix we show explicitly that the metric (1.2) is a solution to Einstein's vacuum field equations. This expression for the family of metrics is given in terms of the family of areal radial functions $\rho_{\lambda}(r)$ which does not violate Birkhoff's theorem since the metric (1.2) expressed in terms of the areal radial functions $\rho_{\lambda}(r)$ has exactly the same functional form as that required by Birkoff's theorem. It is well known to the experts that the extended Schwarzschild metric solution for $r<0$ with $M>0$, corresponds to a solution in the region $r>0$ with $M<0$. Negative masses are associated with repulsive gravity. For this reason, the domain of values of $r$ will be chosen to span the whole real axis $-\infty \leq r \leq \infty$.

The boundary conditions obeyed by the areal radial function $\rho_{\lambda}(r)$ must be at the origin $\rho_{\lambda}(r=0)=0$, and asymptotically $\rho_{\lambda}(r \rightarrow \infty) \sim r \rightarrow \infty$. The Hilbert textbook 
(black hole) solution [5] when $\rho(r)=r$ obeys the boundary conditions but the AbramsBrillouin [4] choice $\rho(r)=r+2 G M$ does not. The original solution of 1916 found by Schwarzschild for $\rho(r)$ did not obey the boundary condition $\rho(r=0)=0$ as well. The condition $\rho(r=0)=2 G M$ has a serious flaw and is : how is it possible for a point-mass at $r=0$ to have a non-zero area $4 \pi(2 G M)^{2}$ and a zero volume simultaneously ?; so it seems that one is forced to choose the Hilbert areal radial function $\rho(r)=r$. It is known that fractals have unusual properties related to their lengths, areas, volumes, dimensions but we are not focusing on fractal spacetimes at the moment. For instance, one could have a fractal horizon surface of infinite area but zero volume (space-filling fractal surface). The finite area of $4 \pi(2 G M)^{2}$ could then be seen as a regularized value of the infinite area of a "fractal horizon".

The Hilbert choice for the areal radial function $\rho(r)=r$ is ultimately linked to the actual form of the Newtonian potential $V_{N}=-\left(G m_{1} m_{2} / r\right)$. In the last few decades corrections to Newton's law of gravitation and constraints on them have become the subject of considerable study, see the monograph [6]. Yukawa-type corrections to Newton's gravitational law from two recent measurements of the Casimir interaction between metallic surfaces was studied by [7]. A Yukawa-like correction to the Newtonian potential could be chosen to be

$$
V(r)=-\frac{G m_{1} m_{2}}{r}\left(1-\lambda e^{-r / 2 G M}\right), \quad \lambda>0
$$

where $\lambda$ and $r_{o}=2 G M$ are the strength and interaction range of the Yukawa-type correction. One may notice that the potential (1.3a) can be rewritten in terms of an areal-radial function $\rho(r)$ as

$$
V(r)=-\frac{G m_{1} m_{2}}{\rho(r)}, \quad \rho(r)=\frac{r}{1-\lambda e^{-r / 2 G M}}, \quad \lambda \neq 1
$$

One has the correct boundary conditions for the areal radial function when $\lambda \neq 1$

$$
\rho(r=0)=0 ; \quad \rho_{\lambda}(r \rightarrow \infty) \rightarrow r, \quad \rho\left(r=r_{h}\right)=2 G M ; \quad 0 \leq r_{h} \leq 2 G M
$$

so that the location of the horizon radius $r_{h}$ has been shifted towards the singularity. In the asymptotic regime one has as expected $\rho(r \rightarrow \infty) \rightarrow r$, so that the areal-radial function tends to $r$ (as in the Hilbert choice) and the expression for the potential is asymptotic to the Newtonian one. At the end of this section we shall discuss the case when $\lambda=1$.

Instead of the Yukawa-type areal radial function (1.3b), one could have had a different one-parameter family of areal-radial functions $\rho_{\lambda}(r)^{1}$. In particular, the metric solutions (1.2) are invariant under the transformations $r \rightarrow-r ; M \rightarrow-M$ for our particular choice of the areal radial functions $\rho_{\lambda}(r)$ given in eq- $(1.3 \mathrm{~b})$ and due to the condition $\rho_{\lambda}(-r,-M)=-\rho_{\lambda}(r, M)$. This allows us to extended the solutions to the $r<0$ region. For a recent analysis of the properties of the maximal extensions ( in regions $r<0$ ) of the Kerr and Kerr-Newman spacetimes with negative mass, see [8].

\footnotetext{
${ }^{1}$ We thank Matej Pavsic for a discussion on other choices for the radial functions
} 
It is also important to emphasize that the Newtonian potential is recovered in general in the regime when $r>>2 G M$, so that $V(r)=-\frac{G m_{1} m_{2}}{\rho(r)} \simeq-\frac{G m_{1} m_{2}}{r}$. For example, in the case of the sun its Schwarzschild radius $2 G M$ is of the order of $3 \mathrm{Kms}$ which is much smaller than the solar radius and the scale of the planetary orbits. Consequently, in the regime when $r>>2 G M$, all the metric solutions in eq-(1.2) reduce to the standard textbook Hilbert solution in eq-(1.1), and the Newtonian potential is always recovered from an infinity of modified potentials.

Conversely, rather than starting from the Hilbert metric solution (1.1), and followed by the active diffeomorphisms $r \rightarrow \rho(r)$ in order to obtain the metric solutions in eq(1.2), one could have started instead with the most general metric solutions in eq-(1.2), and afterwards choose the Hilbert gauge $\rho(r)=r$ leading to the textbook metric solution (1.1) which is associated with the Newtonian potential. In this respect, one could assign a special status to the Hilbert gauge $\rho(r)=r$ since it is the one which leads to the Newtonian potential.

However this does not imply that we have to exclude other possible choices (gauges) for the areal radial function $\rho(r)$. In particular, if one has an equivalence class of metrics $[\mathrm{g}]$, such that $g, g^{\prime}$ belong to the same class, then $g \sim g^{\prime}$ iff $g$ is diffeomorphic to $g^{\prime}$. Why then should we exclude $g^{\prime}$ from the picture and just retain $g$ ? why not exclude $g$ and retain $g^{\prime}$ instead ?. Therefore, all these mathematically distinct solutions obtained via the active diffeomorphisms $r \rightarrow \rho(r)$ are not physically distinguishable, but they represent one and the same physical solution of the field equations. For a historical account of the role of active and passive diffeomorphisms within the context of the "hole argument" that much troubled Einstein we refer to [10].

Consequently, there are two routes one could embark on. (i) One could single out from the beginning the Hilbert metric (1.1) associated to the Newtonian potential. (ii) Or one could choose the metric in eq-(1.2) for any given expression of the areal radial function $\rho(r)$ compatible with the boundary conditions, like the ones displayed in eq- $(1.3 \mathrm{~b})$, and corresponding to the modified Newtonian potentials (1.3a). The use of the metric in eq(1.1) or in eq-(1.2) would be just a matter of taste since both are physically equivalent. In all these cases the metric solutions will no longer be static inside the interior regions $r<2 G M(\rho<2 G M)$. Below we shall explain how to obtain a truly static metric everywhere when the horizon merges with the singularity by choosing the areal radial function given in eq-(1.5).

The Penrose diagrams associated with the solutions described in (1.2) are the same as the diagrams corresponding to the extended Schwarzchild solutions with the only difference that we must replace the radial variable $r$ for $\rho$. The horizons at the radial locations $r_{h}^{(\lambda)}$ all correspond to the unique value of the areal radial function $\rho\left(r_{h}^{(\lambda)}\right)=2 G M$ and $t= \pm \infty$. The spatial singularity is located at $\rho_{\lambda}(r=0)=0$. The Fronsdal-KruskalSzekeres change of coordinates that permit an analytical extension into the interior region of the black hole has the same functional form as before after replacing $r$ for $\rho$. In the exterior region $\rho(r)>2 G M$ one has 


$$
U=\left(\frac{\rho(r)}{2 G M}-1\right)^{\frac{1}{2}} e^{\rho(r) / 4 G M} \cosh \left(\frac{t}{4 G M}\right), \quad V=\left(\frac{\rho(r)}{2 G M}-1\right)^{\frac{1}{2}} e^{\rho(r) / 4 G M} \sinh \left(\frac{t}{4 G M}\right) ;
$$

The change of coordinates in the interior region $\rho(r)<2 G M$ is

$$
U=\left(1-\frac{\rho(r)}{2 G M}\right)^{\frac{1}{2}} e^{\rho(r) / 4 G M} \sinh \left(\frac{t}{4 G M}\right), \quad V=\left(1-\frac{\rho(r)}{2 G M}\right)^{\frac{1}{2}} e^{\rho(r) / 4 G M} \cosh \left(\frac{t}{4 G M}\right)
$$

In the overlap $\rho(r)=2 G M$ region, one has straight lines $U= \pm V$ of $\pm \pi / 4$ slope when $t= \pm \infty$, and $U=V=0$ for finite $t$.

The coordinate transformations lead to a well behaved metric (except at $\rho(r=0)=0$ )

$$
d s^{2}=\frac{4(2 G M)^{3}}{\rho(U, V)} e^{-\rho(U, V) / 2 G M}\left(d V^{2}-d U^{2}\right)-\rho(U, V)^{2}(d \Omega)^{2} .
$$

When $\rho\left(r=r_{\text {horizon }}\right)=2 G M$ and $d \Omega=0$, the above interval displacement $d s^{2}=0$ is null along the lines $U= \pm V \Rightarrow d U= \pm d V$. The interval is singular $d s^{2}=-\infty$ at $\rho(r=0)=0$; i.e. the singularity $r=0$ corresponds to the spacelike lines $V^{2}-U^{2}=$ $1 \Rightarrow d V^{2}-d U^{2}=-d U^{2} /\left(U^{2}+1\right)<0$. A salient feature of the metric $(1.4 \mathrm{c})$ is that is no longer static in the $U, V$ coordinates. This is a reflection of the fact that the roles of $r$ and $t$ are interchanged inside the horizon due to the signature flip when $r<2 G M$ in eq-(1.1), and $\rho_{\lambda}<2 G M$ in eq-(1.2).

In the extreme limiting case $\lambda \rightarrow 1$ any infalling observer reaches a horizon $r_{h}^{(\lambda)}$ whose location approaches arbitrarily close to the singularity $r=0$. To model the scenario when the horizon merges precisely with the singularity one needs an area radial function defined as follows

$$
\rho(r=0)=0, \quad \rho(r)=\frac{r}{1-e^{-r / 2 G M}}, \quad r>0
$$

Under $r \rightarrow-r ; M \rightarrow-M$ one has that $\rho(r) \rightarrow-\rho(r)$ so one can ensure the invariance of the metric (1.2) under these transformations and extend the solutions to the $r<0$ region.

Hence, we have in eq-(1.5) that $\rho\left(r=0^{+} ; M\right)=2 G M$, and $\rho\left(r=0^{-} ;-M\right)=-2 G M$, but $\rho(r=0)=0$ since a point mass must have zero area and zero volume. The horizon is located at $r_{h}=0^{+}$and the singularity at $r=0$. There is a discontinuity of $\rho(r)$ at $r=0$. The right $r=0^{+}$, and left $r=0^{-}$limits of $\rho(r)$ give respectively $\pm 2 G M$, while $\rho(r=0)=0$ which is the arithmetic mean of $2 G M$ and $-2 G M$.

In the Appendix it is shown that the areal-radial function $\rho(r)$ given by eq-(1.5) (along with an infinite number of $C^{\infty}$ differentiable functions) solves the vacuum field equations. The finite discontinuity of $\rho(r)$ occurs at one single point $r=0$ (the origin), whereas $(d \rho / d r)=\infty$ at $r=0^{ \pm}$. Hence, the derivatives of $\rho(r)$ are continuous while $\rho(r)$ is discontinuous at $r=0$. A typical example of this behavior is the tangent function $\tan (\theta)$. At $\theta=\pi / 2$, the tangent exhibits a discontinuity as it goes from $\infty$ to $-\infty$, whereas the derivative remains the same and equal to $\infty$. Fractal curves on the other hand are continuous everywhere but nowhere differentiable. Concluding, the derivatives of $\rho(r)$ 
are continuous, and the metric (1.2) whose areal-radial function $\rho(r)$ is given by eq-(1.5) solves the vacuum field equations as shown in the Appendix.

Because a point mass is an infinitely compact source of infinite density, there is nothing wrong with the possibility of having a discontinuity of the metric at the location of the singularity $r=0$. Due to the boundary condition $\rho(r=0)=0$, there is a curvature tensor singularity and the Kretschmann invariant $R_{\mu \nu \rho \sigma} R^{\mu \nu \rho \sigma} \sim(2 G M)^{2} / \rho(r)^{6}$ diverges at $\rho(r=0)=0$. In this extreme case, when the the location of the horizon merges with the singularity, there is a null-line singularity at $r=0$ and a null-surface at $r=0^{+}$. This may sound quite paradoxical but it is a consequence of the metric discontinuity at $r=0$, the location of the point mass (singularity). This key fact may have important consequences for the resolution of the fire wall problem and the complementarity controversy in black holes [13], [16].

The discontinuity of the metric due to the behavior of the areal-radial function $\rho\left(r=0^{+} ; M\right)=2 G M, \rho(r=0)=0$, can effectively be interpreted as saying that the "point" mass has a finite area but zero volume [11]. It is generally believed that any quantum theory of gravity should have a generic feature, a quantum of length, which can be taken to coincide with the Planck scale. If the appropriately defined effective dimension, $D_{\text {eff }}$, decreases continuously from $D_{\text {eff }}=D$ at very large distances compared to the Planck scale, down to $D_{\text {eff }}=2$ at the Planck scale, this suggests that the physical spacetime becomes essentially 2-dimensional near Planck scale. For a detailed study of this phenomenon we refer to [11].

When the areal-radial function $\rho(r)$ has the actual form in eq-(1.5) there is no interior region beyond the horizon $r=0^{+}$, so that the metric (1.2) is truly static everywhere. The Fronsdal-Kruskal-Szekeres analytical continuation of the metric (1.4c) inside the horizon is not static. 't Hooft [14] has most recently argued how black hole unitarity demands the existence of transformations that can remove firewalls at the standard horizon radius $2 G M$. A continuity condition is imposed with an antipodal identification as an inevitable consequence. 't Hooft argued that it is necessary to revise the boundary conditions (and topology) for Nature's degrees of freedom at the horizon of a black hole. The boundary condition is characterized as an antipodal identification and what it means is that the region of space-time inside the horizon is removed completely, as if by surgery, after which the edges are glued together by identifying the antipodes.

Klinkhamer [15] provided earlier on a regularization of the standard Schwarzschild solution with a curvature singularity at the center by removing the interior region of a ball and identifying the antipodal points on the boundary. The resulting four-dim manifold has now the topology $R \times \tilde{M}_{3}$ where $\tilde{M}_{3}$ is a nonsimply-connected manifold, which up to a point (the center), is homeomorphic to the 3 -dim real projective space $R P^{3}$. In our case, there is no need to remove the interior region by surgery. The discontinuity $\rho(r=0)=0 ; \rho\left(r=0^{+}\right)=2 G M$ of the areal-radial function amounts to a sort of "pointsplitting" creating a void (hole) in spacetime, isolating and expunging the curvature singularity at the center from the remaining region of spacetime. The topology of the region free of the singularity at the center is $\tilde{M}_{4}=R \times \tilde{M}_{3}$, where $\tilde{M}_{3}=R^{3}-\{0\}$ is the punctured 3-dim space. After this introductory review one may proceed. 


\section{Renormalization Group Improved Einstein's Equa- tions}

The Renormalization Group (RG) improvement of Einstein's equations is based on the possibility that Quantum Einstein Gravity might be non-perturbatively renormalizable and asymptotically safe due to the presence of interacting ultraviolet fixed points [17]. In this program one has $k$ (energy) dependent modifications to the Newtonian coupling $G(k)$, the cosmological constant $\Lambda(k)$ and energy-dependent spacetime metrics $g_{i j,(k)}(x)$. The purpose of this section is to establish a one-to-one correspondence between the renormalization group flow of the Newtonian coupling $G(k)$ and the diffeomorphism flows $r \rightarrow \rho(r)$ of the spacetime points.

Quantum gravitational effects in spherically symmetric black hole spacetimes were studied by [18]. The effective quantum spacetime felt by a point-like test mass was constructed by "Renormalization Group improving" the Schwarzschild metric. The key ingredient is the running Newton constant which is obtained from the exact evolution equation for the effective average action. As a consequence of the quantum effects, the classical singularity at $r=0$ was either removed completely or was at least much milder than classically. The Asymptotic safety program has also found important applications in Fractals and Cosmology [19] hinting at the possibility that a fractal spacetime arises at Planck scales, this picture was also envisioned by Nottale long ago in his formulation of Scale Relativity Theory [20].

In $D=4$ there is a nontrivial interacting (non-Gaussian) ultraviolet fixed point $G_{*}=G(k) k^{2} \neq 0$. The fixed point $G_{*}$ by definition is dimensionless and the running gravitational coupling has the form [18], [17]

$$
G(k)=G_{N} \frac{1}{1+\left[G_{N} k^{2} / G_{*}\right]}
$$

The Renormalization Group (RG) improvement of the Schwarzschild metric components are defined to be $[18]$

$$
g_{00, k}(r)=1-\frac{2 G(k) M}{r}, \quad g_{r r, k}(r, E)=-\left(1-\frac{2 G(k) M}{r}\right)^{-1}
$$

however if one assigns an energy-scale $k \leftrightarrow r$ correspondence by introducing a nontrivial functional relation of the form $k=k(r)$, and substitutes back its expression into the scale-dependent metric components (2.2) one will no longer satisfy the vacuum Einstein field equations (which led to the Schwarzschild solutions in the first place)

$$
R_{\mu \nu}-\frac{1}{2} g_{\mu \nu} R=0
$$

consequently, one will be forced to modify Einstein's equations. For example, via the introduction of $f(R, \nabla R)$ Lagrangians into the modified gravitational action involving polynomials in the curvature tensor and its covariant derivatives, or more complicated functions $f(R, \nabla R)$. 
For this reason, it is far simpler to exploit the more general solutions (1.2) to the vacuum field equations which are diffeomorphic but not isometric to the Schwarzschild solution (1.1). Therefore, one may define the $k \leftrightarrow r$ correspondence by absorbing the energy dependent (running) gravitational coupling $G(k)$ into the definition of the arealradial function $\rho(r)$ introduced in the metric (1.2). This is achieved by writing eq- $(2.2)$ (for $r>0$ ) in the form

$$
\begin{gathered}
1-\frac{2 G(k(r)) M}{r}=1-\frac{2 G_{N} M}{\rho(r)} \Rightarrow \\
\frac{G(k(r))}{r}=\frac{G_{N}}{\rho(r)} \Rightarrow G_{N} \frac{1}{1+\left[G_{N} k^{2}(r) / G_{*}\right]}=G_{N} \frac{r}{\rho(r)}, \quad r>0
\end{gathered}
$$

and in this fashion one recasts the two metric components (2.2) as two pieces of the most general solutions displayed by eq-(1.2) involving the areal-radial function $\rho(r)$ given by eq-(1.5). ${ }^{2}$ Hence, from eq-(2.4) and eq-(1.5) one can read-off directly the sought-after $k \leftrightarrow r$ correspondence

$$
k^{2}(r)=\frac{G_{*}}{G_{N}} \frac{e^{-r / 2 G_{N} M}}{1-e^{-r / 2 G_{N} M}}
$$

the most salient feature of the energy-scale relation eq-(2.5) is that it does not have the same functional dependence as obtained in [18] and given by $k \sim 1 / d(r)$ where $d(r)$ is the proper radial distance between two points $\int_{r_{o}}^{r} \sqrt{\left|g_{r r}\right|} d r$. It was emphasized earlier that metrics of the type

$$
(d s)^{2}=\left(1-\frac{2 G(k(r)) M}{r}\right)(d t)^{2}-\left(1-\frac{2 G(k(r)) M}{r}\right)^{-1}(d r)^{2}-r^{2}(d \Omega)^{2} .
$$

do not solve the vacuum Einstein field equations.

Before proceeding let us mention that one could try to remedy this problem by adding matter sources, corresponding to the stress energy tensor $T_{\nu}^{\mu}=\left(\sigma,-p_{r},-p_{\theta},-p_{\phi}\right)$, to the right hand side of Einstein equations, and associated to a mass source distribution of the form $M(r)=\int_{0}^{r} \sigma\left(r^{\prime}\right) 4 \pi r^{\prime 2} d r^{\prime}$. The expression for $M(r)$ is obtained from the identification $G(k(r)) M \equiv G_{N} M(r)$. The conservation of the stress energy tensor $\nabla_{\mu} T_{\nu}^{\mu}=0$ imposes additional relations among the components in $\left(\sigma,-p_{r},-p_{\theta},-p_{\phi}\right)$. We refer to the review work by [12] where, in particular, a self gravitating droplet with anisotropic pressure was taken as the source. It is based on smearing a point mass delta function distribution by introducing a Gaussian mass density $\sigma(r)$ of finite width and which determined the functional form of $M(r)$ in terms of an incomplete Euler gamma function. We shall not pursue this route, just fix $M$ to a constant and focus on the vacuum solutions only.

Given $\rho\left(r=0^{+}\right)=2 G_{N} M$, the above expression for $k^{2}(r)$ in eq- $(2.5)$ has the following properties :

\footnotetext{
${ }^{2}$ One must exclude $r=0$ in eq- $(2.4)$ due to the requirement $\rho(r=0)=0$, and $\rho\left(r=0^{+}\right)=2 G_{N} M$ resulting from the discontinuity of the metric at $r=0$
} 
(i) when $r \rightarrow 0^{+}, k \rightarrow \infty$, one recovers the expected ultraviolet limit at infinitesimally small distances approaching zero. i.e. it will take an infinite energy to probe $r=0^{+}$.

(ii) when $r \rightarrow \infty, k \rightarrow 0$, one recovers the expect infrared limit at infinite large distances.

(iii) when $\frac{r}{2 G_{N} M}<<1 \Rightarrow k^{2} \sim\left(2 G_{*} M / r\right)$; i.e. when $r$ is much smaller than the standard black hole horizon radius $2 G_{N} M$, one arrives at an energy-scale dependence of the form $k \sim r^{-1 / 2}$. This behavior should be compared with the "naive" dependence $k \sim 1 / r$.

(iv) At Planck scales $r \sim L_{P}$, the condition (iii) implies $M>>m_{P}$, which in turn leads to $k^{2} \sim M m_{P}>>m_{P}^{2}$, so the energy $k \sim \sqrt{M m_{P}}$ required to explore the Planck scale size regions would be much higher than the Planck mass.

(v) Had one chosen an areal radial function of the form $\rho_{\lambda}(r)=r\left(1-\lambda e^{-r / 2 G_{N} M}\right)^{-1}, \lambda \neq 1$, the value of $k^{2}$ as $r \rightarrow 0$, is $\frac{G_{*} \lambda}{G_{N}(1-\lambda)} \neq \infty$ and would no longer be infinite. This is not compatible with the ultraviolet completion program of asymptotic safety.

(vi) The choice $k=1 / r$ is associated to an areal-radial function $\rho(r)$ which is derived from the equality

$$
G_{N} \frac{1}{1+\left[G_{N} k^{2}(r) / G_{*}\right]}=G_{N} \frac{1}{1+\left[G_{N} / G_{*} r^{2}\right]}=G_{N} \frac{r}{\rho(r)}
$$

and leading to

$$
\rho(r)=r+\frac{G_{N}}{G_{*} r}
$$

the above expression for $\rho(r)$ has the correct behavior at large distances $\rho(r) \sim r$ but it blows up at $r=0$. Consequently, it does not have the correct boundary condition $\rho(r=0)=0$. The functional form (2.8) is reminiscent of the minimal length modified stringy uncertainty relations since $\rho(r)$ defined by eq-(2.8) has a minimum value of $\rho_{\text {min }}=$ $2 \sqrt{\left(G_{N} / G_{*}\right)}$ at $r_{*}=\sqrt{\left(G_{N} / G_{*}\right)}$.

The construction presented here can also be extended to metrics which are are diffeomorphic but not isometric to the (Anti) de Sitter-Schwarzschild metrics

$$
\begin{gathered}
(d s)^{2}=\left(1-\frac{2 G_{N} M}{\rho(r)}-\frac{\Lambda_{0}}{3} \rho(r)^{2}\right)(d t)^{2}-\left(1-\frac{2 G_{N} M}{\rho(r)}-\frac{\Lambda_{0}}{3} \rho(r)^{2}\right)^{-1}(d \rho(r))^{2}- \\
(\rho(r))^{2}(d \Omega)^{2}
\end{gathered}
$$

$\Lambda_{0}>0$ for de Sitter space and $\Lambda_{0}<0$ for Anti de Sitter. By writing (for $r>0$ )

$$
1-\frac{2 G(k(r)) M}{r}-\frac{\Lambda(k(r))}{3} r^{2}=1-\frac{2 G_{N} M}{\rho(r)}-\frac{\Lambda_{0}}{3} \rho(r)^{2}, \quad r>0
$$

and establishing a $k \leftrightarrow r$ correspondence one can incorporate the Renormalization Group flow of the coupling $G(k(r))$ and the running cosmological constant $\Lambda(k(r))$ into the arealradial function $\rho(r)$ in the right hand side of (2.10). The scale dependence of $\Lambda(k)$ in the de Sitter case was found to be [18] 


$$
\Lambda(k)=\Lambda_{0}+\frac{b G(k)}{4} k^{4}, \quad \Lambda_{0}>0
$$

where $b$ is positive numerical constant. In $D=4$, the dimensionless gravitational coupling has a nontrivial fixed point $G=G(k) k^{2} \rightarrow G_{*}$ in the $k \rightarrow \infty$ limit, and the dimensionless variable $\Lambda=\Lambda(k) k^{-2}$ has also a nontrivial ultraviolet fixed point $\Lambda_{*} \neq 0$ [18]. The infrared limits are $\Lambda(k \rightarrow 0)=\Lambda_{0}>0, G(k \rightarrow 0)=G_{N}$. Whereas the ultraviolet limit is $\Lambda(k=\infty)=\infty ; G(k=\infty)=0$.

Upon substituting the expressions for $G(k), \Lambda(k)(2.1,2.11)$ into the left hand side of eq-(2.10), it furnishes a new energy-scale relation $k(r)$ in terms of the given areal-radial function $\rho(r)$ of eq-(1.5) appearing in the right hand side of eq-(2.12). The functional relation is now given by the solutions of a quadratic equation

$$
k^{2}(r)=\frac{-B+\sqrt{B^{2}-4 A C}}{2 A}
$$

where we chose the plus sign in front of the square root to ensure $k^{2}(r) \geq 0$. The expressions for $A, B, C$ are respectively

$$
\begin{gathered}
A(r) \equiv \frac{b G_{N}}{12} r^{2}>0, \quad B(r) \equiv \frac{G_{N}}{G_{*}}\left(\frac{\Lambda_{0}}{3}\left(\rho(r)^{2}-r^{2}\right)+\frac{2 G_{N} M}{\rho(r)}\right)>0 \\
C(r) \equiv 2 G_{N} M\left(\frac{1}{\rho(r)}-\frac{1}{r}\right)+\frac{\Lambda_{0}}{3}\left(\rho(r)^{2}-r^{2}\right)
\end{gathered}
$$

Due to the more complicated functional expression for $k^{2}(r)$ in eq- $(2.12 \mathrm{a})$ one has to ensure that $k^{2}(r) \geq 0$ for $r>0$. Since $\Lambda_{0}>0$, and the areal radial function $\rho(r)=$ $r\left(1-e^{-r / 2 G_{N} M}\right)^{-1} \geq r$, the values of $C(r)$ in eq-(2.12b) will cease to be negative in the crossover region determined by the value of $r=r_{c}$ such that $C\left(r_{c}\right)=0$. Consequently, in the region $r>r_{c}, C(r)>0$, the values of $k^{2}(r)<0$ will become negative which are unphysical. $k^{2}(r)$ becomes 0 at $r=r_{c}$ and $r=\infty$.

Before deriving the value of the crossover point $r_{c}$, a careful inspection of eqs- $(2.12 \mathrm{a}$, $2.12 \mathrm{~b})$ based on the graph of the areal-radial function in the region $r>0, \rho(r)=r(1-$ $\left.e^{-r / 2 G_{N} M}\right)^{-1} \geq r$, reveals that $k^{2}(r \rightarrow \infty) \rightarrow 0$, and $k^{2}\left(r \rightarrow 0^{+}\right) \rightarrow \infty$ as expected.

When $r \rightarrow 0^{+} \Rightarrow 4 A C \rightarrow 0$, and one can perform the binomial expansion in

$$
\begin{gathered}
k^{2}=\frac{-B+\sqrt{B^{2}-4 A C}}{2 A}=\frac{-B+B \sqrt{1-4 A C / B^{2}}}{2 A} \sim \\
\frac{-B+B\left(1-2 A C / B^{2}\right)}{2 A}=\frac{-C}{B}
\end{gathered}
$$

Hence, in the limit $r \rightarrow 0^{+}$, given that $\rho\left(r=0^{+}\right)=2 G_{N} M$, eq- $(2.13)$ yields

$$
k^{2}\left(r \rightarrow 0^{+}\right) \rightarrow \lim _{r \rightarrow 0^{+}} \frac{-C}{B} \rightarrow
$$




$$
\lim _{r \rightarrow 0^{+}} \frac{\left(2 G_{N} M / r\right)}{\left(G_{N} / G_{*}\right)\left(1+\left(\Lambda_{0} / 3\right)\left(2 G_{N} M\right)^{2}\right)}=\lim _{r \rightarrow 0^{+}} \beta \frac{G_{*} M}{r} \rightarrow \infty
$$

therefore, from eq- $(2.14)$ one can infer that the ultraviolet scaling is of the form

$$
k^{2} r \sim \beta\left(G_{*} M\right), \quad \beta \equiv \frac{2}{1+\left(\Lambda_{0} / 3\right)\left(2 G_{N} M\right)^{2}}, \Lambda_{0}>0
$$

In the infrared limit $r \rightarrow \infty, A \rightarrow \infty, B \rightarrow 0, C \rightarrow 0, \rho(r) \sim r$, one has trivially $k^{2} \rightarrow 0$.

It was not necessary to solve for $k^{2}(r)$ in eq-(2.10) in order to glean the infrared and ultraviolet behavior. One can infer from the ultraviolet scaling behavior, given by $k^{2} r \sim \beta G_{*} M,(\beta \neq 2)$ when $r \rightarrow 0^{+}, k \rightarrow \infty$, that the products $k^{2} r ; k^{4} r^{2}$ remain finite so the left and right hand sides of eq- $(2.10)$ coincide $\left(\rho\left(r=0^{+}\right)=2 G_{N} M\right)$. Conversely, the infrared scaling behavior is $k^{4} r^{2} \rightarrow 0$, when $r \rightarrow \infty, k \rightarrow 0, \rho(r) \sim r$, such that eq- $(2.10)$ holds given that $G(k=0)=G_{N}, \Lambda(k=0)=\Lambda_{0}$.

Lets determine the value of the crossover point $r_{c}$ that forces $k^{2}\left(r>r_{c}\right)<0$ to be negative. It is determined from the solution to the transcendental equation

$$
\frac{\Lambda_{0}}{3} \frac{\left(2-e^{-r_{c} / 2 G_{N} M}\right)}{\left(1-e^{-r_{c} / 2 G_{N} M}\right)^{2}} r_{c}^{2}-\frac{2 G_{N} M}{r_{c}}=0
$$

The transcendental equation (2.16) is greatly simplified when $r_{c}>>2 G_{N} M$. Given $\frac{\Lambda_{0}}{3}=\frac{1}{R_{H}^{2}}\left(R_{H}=\right.$ Hubble radius $)$, eq-(2.16) leads to

$$
\frac{2 \Lambda_{0}}{3} r_{c}^{2}-\frac{2 G_{N} M}{r_{c}} \simeq 0 \Rightarrow r_{c} \simeq 2^{-\frac{1}{3}}\left[\left(2 G_{N} M\right) R_{H}^{2}\right]^{\frac{1}{3}}, \frac{\Lambda_{0}}{3}=\frac{1}{R_{H}^{2}}
$$

so that a simple solution $r_{c}$ given by (2.17a) is found in the regime when

$$
2 G_{N} M<<r_{c}<<R_{H}
$$

To sum up, the use of the areal-radial function $\rho(r)$ of eq-(1.5) in eq-(2.10), for $r>0$, does not lead to a fully satisfactory energy-scale $k(r)$ functional relation due to the fact that $k^{2}(r)<0$ when $r>r_{c}$, despite that $k^{2}\left(r \rightarrow 0^{+}\right) \rightarrow \infty$, and $k^{2}(r \rightarrow \infty) \rightarrow 0$. What this entails is that one cannot accommodate two running couplings $G(k(r)), \Lambda(k(r))$, for all values of $r>0$, within one single function : the areal-radial function $\rho(r)$. The analysis of this section can be generalized to higher dimensions.

To finalize this section we should mention that it was pointed out by Donoghue [14] that the standard perturbative quantization of Einstein gravity leads to a well-defined, finite prediction for the leading large distance correction to Newton's gravitational potential energy, after restoring $\hbar$ and $c$, given by

$$
V(r)=-G_{N} \frac{M m}{r}\left(1-\frac{G_{N}(M+m)}{2 c^{2} r}-\frac{\alpha G_{N} \hbar}{c^{3} r^{2}}\right), \quad \alpha=\frac{118}{15 \pi}
$$

the first correction proportional to $\frac{(M+m)}{r}$ is a purely kinematic effect of classical general relativity, while the second correction proportional to $1 / r^{2}$ is a quantum effect. 
The modified potential energy (2.14) can also be rewritten in terms of another areal function $\tilde{\rho}(r)$ differing from eq-(1.5), as

$$
V(r)=-G_{N} \frac{M m}{\tilde{\rho}(r)}, \quad \tilde{\rho}(r) \equiv \frac{r}{1-G_{N}(M+m) / 2 c^{2} r-\alpha G_{N} \hbar / c^{3} r^{2}}
$$

the new areal-radial function does obey the required boundary conditions described in section 1. $\tilde{\rho}(r=0)=0$; and asymptotically $\tilde{\rho}(r) \sim r$. Furthermore, when $r \rightarrow-r$, and $(M, m) \rightarrow(-M,-m)$ we have $\tilde{\rho}(r) \rightarrow-\tilde{\rho}(r)$ so that a metric of the form (1.2) remains invariant and can be extended to the region $r<0$.

\section{Asymptotic Safety and the Geometry of Phase Space}

The Lagrange-Finsler geometry of the tangent bundle (Hamilton-Cartan geometry of the cotangent bundle) of spacetimes has been the subject of intensive study in recent years [22], [23]. It is better understood now that the Planck-scale modifications of the particle dispersion relations can be encoded in the nontrivial geometrical properties of momentum space [24]. When both spacetime curvature and Planck-scale deformations of momentum space are present, it is expected that the nontrivial geometry of momentum space and spacetime get intertwined. The interplay between spacetime curvature and non-trivial momentum space effects was essential in the notion of "relative locality" and in the deepening of the relativity principle [24].

The authors [25], [26] have described the Hamilton geometry of the phase space of particles whose motion is characterized by general dispersion relations. Explicit examples of two models for Planck-scale modified dispersion relations, inspired from the $q$-de Sitter and $\kappa$-Poincare quantum groups, were considered. In the first case they found the expressions for the momentum and position dependent curvature of spacetime and momentum space, while for the second case the manifold is flat and only the momentum space possesses a nonzero, momentum dependent curvature.

Starting with the geometry of the cotangent bundle (phase space), it was shown in [27] that the maximal proper force condition, in the case of a uniformly accelerated observer of mass $m$ along the $x$ axis, leads to a minimum value of $x$ lying inside the Rindler wedge and given by the black hole horizon radius $2 \mathrm{Gm}$. Whereas in the uniform circular motion case, the maximal proper force condition implied that the radius of the circle cannot exceed the value of the horizon radius $2 \mathrm{Gm}$. A correspondence was found between the black hole horizon radius and a singularity in the curvature of momentum space. In this final section we argue how the Asymptotic Safety program in gravity [17], [18], combined with the phase space geometry seem to be a proper arena for a space-time-matter unification.

The $8 D$ cotangent space/phase-space associated to a $4 D$ spacetime has for coordinates $\mathbf{x}, \mathbf{p}$ and the infinitesimal interval in phase space is 


$$
(d \sigma)^{2}=g_{i j}(\mathbf{x}, \mathbf{p}) d x^{i} d x^{j}+h_{a b}(\mathbf{x}, \mathbf{p})\left(d p^{a}-N_{i}^{a}(\mathbf{x}, \mathbf{p}) d x^{i}\right)\left(d p^{b}-N_{j}^{b}(\mathbf{x}, \mathbf{p}) d x^{j}\right)
$$

where the $N$-coefficients define a nonlinear connection, an $N$-connection structure. For rigorous details we refer to [22], [23]. In a very particular case one recovers the ordinary linear connection if $N_{i}^{a}(\mathbf{x}, \mathbf{p})=\Gamma_{b i}^{a}(\mathbf{x}) p^{b}$.

The vacuum field equations were given by [23]

$$
\begin{aligned}
& \mathcal{R}_{i j}-\frac{1}{2}(\mathcal{R}+\mathcal{S}) g_{i j}=0, \quad i, j=1,2,3,4 . \\
& \mathcal{S}_{a b}-\frac{1}{2}(\mathcal{R}+\mathcal{S}) h_{a b}=0, \quad a, b=1,2,3,4 .
\end{aligned}
$$

where $\mathcal{R}_{i j}, \mathcal{R}$ and $\mathcal{S}_{a b}, \mathcal{S}$ are the Ricci and scalar curvatures of spacetime and the internal momentum space, respectively. The indices $i, j$ are the horizontal base space indices, while $a, b$ are the vertical space ones corresponding to the fibers. The geometry of the cotangent bundle is very intricate and it involves six distinguished curvature tensors, five distinguished torsion tensors and the nonlinear connections components [22], [23]. Hence, to find exact solutions to the very intricate set of eqs- $(3.2,3.3)$ is highly nontrivial [23], $[28]$.

The additional momentum-dependent behavior of the field equations $(3.2,3.3)$ (beside the $\mathbf{x}$-dependence) could cast more light into the study of the Renormalization Group (RG) improvement of Einstein's equations which was based on the possibility that Quantum Einstein Gravity might be non-perturbatively renormalizable and asymptotically safe due to the presence of interacting ultraviolet fixed points [17]. As seen in the prior section, one has $k$ (energy) dependent modifications to the Newtonian coupling $G(k)$, the cosmological constant $\Lambda(k)$ and energy dependent spacetime metrics $g_{i j,(k)}(x)$. Namely, what one is aiming for is in establishing a quantum spacetime geometry/classical phase geometry correspondence $g_{i j,(k)}(x) \leftrightarrow g_{i j}(x, E)$.

Let us insert directly the energy dependence of $G(k)$ of eq-(2.1) into the components associated to the Schwarzschild metric, by setting $E=k$ and without imposing any energy-distance relationship $k=k(r)$ as done previously. The components of the spacetime metric are now given directly in terms of $r$ and $E$ (there is no $E(r)$ relation)

$$
g_{t t}(r, E)=1-\frac{2 G(E) M}{r}, g_{r r}(r, E)=-\left(g_{t t}(r, E)\right)^{-1}
$$

The angular part can be chosen to be $r^{2}(d \Omega)^{2}$ as usual. The task now is to find the expressions (if possible) for the internal momentum space metric $h_{a b}(\mathbf{x}, \mathbf{p})$, and the nonlinear connection $N(\mathbf{x}, \mathbf{p})$ components, such that the above vacuum field equations $(3.2,3.3)$ are satisfied when the spacetime metric components $g_{i j}(\mathbf{x}, \mathbf{p})$ are given by eq-(3.4). There is no guarantee a priori that exact solutions of eqs- $(3.2,3.3)$ of this type exist, nevertheless it is worthy of exploration.

To conclude, related to establishing a quantum geometry/classical phase correspondence $g_{i j,(k)}(x) \leftrightarrow g_{i j}(x, E)$, we should recall that in the Deformation Quantization program there is a one-to-one correspondence between operators in a Hilbert space and functions in a classical phase space $A(x, p)$ subject to a noncommutative (Moyal, Fedosov, 
Kontsevich, ....) star product $A(x, p) * B(x, p)$. Noncommutative and Nonassociative star products were constructed by studying non-geometric string (M-theory) backgrounds with fluxes. These novel star products and their implications to Nonassociative Gravity are currently under intense investigation. In particular, they might provide important clues in order to solve the membrane quantization problem, see [29] and references therein. It is warranted to explore the connection between the asymptotic safety program associated to the path integral quantization procedure in quantum gravity and deformation quantization via the geometry of the cotangent bundle (phase space) of spacetime.

\section{APPENDIX A : Schwarzschild-like solutions in $D>3$}

In this Appendix we follow closely the calculations of the static spherically symmetric vacuum solutions to Einstein's equations in any dimension $D>3$. Let us start with the line element with signature $(-,+,+,+, \ldots,+)$

$$
d s^{2}=-e^{\mu(r)}(d t)^{2}+e^{\nu(r)}(d r)^{2}+R^{2}(r) \tilde{g}_{i j} d \xi^{i} d \xi^{j} .
$$

where the areal radial function $\rho(r)$ is now denoted by $R(r)$ and which must not be confused with the scalar curvature $\mathcal{R}$. Here, the metric $\tilde{g}_{i j}$ corresponds to a homogeneous space and $i, j=3,4, \ldots, D-2$ and the temporal and radial indices are denoted by 1,2 respectively. In our text we denoted the temporal index by 0 . The only non-vanishing Christoffel symbols are given in terms of the following partial derivatives with respect to the $r$ variable and denoted with a prime

$$
\begin{array}{lll}
\Gamma_{21}^{1}=\frac{1}{2} \mu^{\prime}, & \Gamma_{22}^{2}=\frac{1}{2} \nu^{\prime}, & \Gamma_{11}^{2}=\frac{1}{2} \mu^{\prime} e^{\mu-\nu}, \\
\Gamma_{i j}^{2}=-e^{-\nu} R R^{\prime} \tilde{g}_{i j}, & \Gamma_{2 j}^{i}=\frac{R^{\prime}}{R} \delta_{j}^{i}, & \Gamma_{j k}^{i}=\tilde{\Gamma}_{j k}^{i},
\end{array}
$$

and the only nonvanishing Riemann tensor are

$$
\begin{array}{ll}
\mathcal{R}_{212}^{1}=-\frac{1}{2} \mu^{\prime \prime}-\frac{1}{4} \mu^{\prime 2}+\frac{1}{4} \nu^{\prime} \mu^{\prime}, & \mathcal{R}_{i 1 j}^{1}=-\frac{1}{2} \mu^{\prime} e^{-\nu} R R^{\prime} \tilde{g}_{i j}, \\
\mathcal{R}_{121}^{2}=e^{\mu-\nu}\left(\frac{1}{2} \mu^{\prime \prime}+\frac{1}{4} \mu^{\prime 2}-\frac{1}{4} \nu^{\prime} \mu^{\prime}\right), & \mathcal{R}_{i 2 j}^{2}=e^{-\nu}\left(\frac{1}{2} \nu^{\prime} R R^{\prime}-R R^{\prime \prime}\right) \tilde{g}_{i j}, \\
\mathcal{R}_{j k l}^{i}=\tilde{R}_{j k l}^{i}-R^{\prime 2} e^{-\nu}\left(\delta_{k}^{i} \tilde{g}_{j l}-\delta_{l}^{i} \tilde{g}_{j k}\right) . &
\end{array}
$$

The vacuum field equations are

$$
\begin{gathered}
\mathcal{R}_{11}=e^{\mu-\nu}\left(\frac{1}{2} \mu^{\prime \prime}+\frac{1}{4} \mu^{\prime 2}-\frac{1}{4} \mu^{\prime} \nu^{\prime}+\frac{(D-2)}{2} \mu^{\prime} \frac{R^{\prime}}{R}\right)=0, \\
\mathcal{R}_{22}=-\frac{1}{2} \mu^{\prime \prime}-\frac{1}{4} \mu^{\prime 2}+\frac{1}{4} \mu^{\prime} \nu^{\prime}+(D-2)\left(\frac{1}{2} \nu^{\prime} \frac{R^{\prime}}{R}-\frac{R^{\prime \prime}}{R}\right)=0,
\end{gathered}
$$

and

$$
\mathcal{R}_{i j}=\frac{e^{-\nu}}{R^{2}}\left(\frac{1}{2}\left(\nu^{\prime}-\mu^{\prime}\right) R R^{\prime}-R R^{\prime \prime}-(D-3) R^{\prime 2}\right) \tilde{g}_{i j}+\frac{k}{R^{2}}(D-3) \tilde{g}_{i j}=0
$$


where $k= \pm 1$, depending if $\tilde{g}_{i j}$ refers to positive or negative curvature. From the combination $e^{-\mu+\nu} \mathcal{R}_{11}+\mathcal{R}_{22}=0$ we get

$$
\mu^{\prime}+\nu^{\prime}=\frac{2 R^{\prime \prime}}{R^{\prime}}
$$

The solution of this equation is

$$
\mu+\nu=\ln R^{\prime 2}+C,
$$

where $C$ is an integration constant that one sets to zero if one wishes to recover the flat Minkowski spacetime metric in spherical coordinates in the asymptotic region $r \rightarrow \infty$.

Substituting (A.7) into the equation (A.6) we find

$$
e^{-\nu}\left(\nu^{\prime} R R^{\prime}-2 R R^{\prime \prime}-(D-3) R^{\prime 2}\right)=-k(D-3)
$$

or

$$
\gamma^{\prime} R R^{\prime}+2 \gamma R R^{\prime \prime}+(D-3) \gamma R^{\prime 2}=k(D-3)
$$

where

$$
\gamma=e^{-\nu}
$$

The solution of (A.10) for an ordinary D-dim spacetime ( one temporal dimension ) corresponding to a $D-2$-dim sphere for the homogeneous space can be written as

$$
\begin{gathered}
\gamma=\left(1-\frac{16 \pi G_{D} M}{(D-2) \Omega_{D-2} R^{D-3}}\right)\left(\frac{d R}{d r}\right)^{-2} \Rightarrow \\
g_{r r}=e^{\nu}=\left(1-\frac{16 \pi G_{D} M}{(D-2) \Omega_{D-2} R^{D-3}}\right)^{-1}\left(\frac{d R}{d r}\right)^{2} .
\end{gathered}
$$

where $\Omega_{D-2}$ is the appropriate solid angle in $D-2$-dim and $G_{D}$ is the $D$-dim gravitational constant whose units are $(\text { length })^{D-2}$. Thus $G_{D} M$ has units of $(\text { length })^{D-3}$ as it should. When $D=4$ as a result that the 2-dim solid angle is $\Omega_{2}=4 \pi$ one recovers from eq-(A.12) the 4-dim Schwarzchild solution. The solution in eq-(A.12) is consistent with Gauss law and Poisson's equation in $D-1$ spatial dimensions obtained in the Newtonian limit.

For the most general case of the $D$-2-dim homogeneous space we should write

$$
-\nu=\ln \left(k-\frac{\beta_{D} G_{D} M}{R^{D-3}}\right)-2 \ln R^{\prime}
$$

$\beta_{D}$ is a constant equal to $16 \pi /(D-2) \Omega_{D-2}$, where $\Omega_{D-2}$ is the solid angle in the $D-2$ transverse dimensions to $r, t$ and is given by $2 \pi^{(D-1) / 2} / \Gamma[(D-1) / 2]$.

Thus, according to (A.8) we get

$$
\mu=\ln \left(k-\frac{\beta_{D} G_{D} M}{R^{D-3}}\right)+\text { constant } .
$$

we can set the constant to zero, and this means the line element (A.1) can be written as 


$$
\begin{gathered}
d s^{2}=-\left(k-\frac{\beta_{D} G_{D} M}{R^{D-3}}\right)(d t)^{2}+\frac{(d R / d r)^{2}}{\left(k-\frac{\beta_{D} G_{D} M}{R^{D-3}}\right)}(d r)^{2}+R^{2}(r) \tilde{g}_{i j} d \xi^{i} d \xi^{j}= \\
-\left(k-\frac{\beta_{D} G_{D} M}{R^{D-3}}\right)(d t)^{2}+\frac{1}{\left(k-\frac{\beta_{D} G_{D} M}{R^{D-3}}\right)}(d R)^{2}+R^{2}(r) \tilde{g}_{i j} d \xi^{i} d \xi^{j}
\end{gathered}
$$

One can verify, that the equations (A.4)-(A.6), leading to eqs-(A.9)-(A.10), do not determine the form $R(r)$. It is also interesting to observe that the only effect of the homogeneous metric $\tilde{g}_{i j}$ is reflected in the $k= \pm 1$ parameter, associated with a positive (negative) constant scalar curvature of the homogeneous $D-2$-dim space. $k=0$ corresponds to a spatially flat $D-2$-dim section. The metric solution in eq-(1.2) is associated to a different signature than the one chosen in this Appendix, and corresponds to $D=4$ and $k=1$.

\section{Acknowledgments}

We are indebted to M. Bowers for assistance.

\section{References}

[1] A. Einstein, Sitzungsber Preuss Akad Berlin II, 831 (1915).

[2] K. Schwarzschild, Sitzungsber Preuss Akad Berlin I,189 (1916); English translation by S. Antoci and A. Loinger can be found in physics/9905030.

[3] C. Castro, "Novel Remarks on Point Mass Sources, Firewalls, Null Singularities and Gravitational Entropy" Foundations of Physics 46 (2016) 14.

[4] M. Brillouin, Jour. Phys. Rad 23, 43 (1923); English translation by S. Antoci can be found at physics/0002009.

[5] D. Hilbert, Nachr. Ges. Wiss Gottingen Math. Phys K1, 53 (1917); H. Weyl, Ann. Physik (Leipzig) 54, 117 (1917); J. Droste, Proc. Ned. Akad. West Ser. A 19, 197 (1917).

[6] E. Fischbach and C. L. Talmadge, The Search for Non-Newtonian Gravity (Springer, New York, 1999).

[7] G. L. Klimchitskaya, U. Mohideen and V. M. Mostepanenko, "Constraints on corrections to Newtonian gravity from two recent measurements of the Casimir interaction between metallic surfaces", arXiv : 1306.4979.

[8] V. Manko, "On the Properties of Exact Solutions Endowed with Negative Mass", arXiv : 1303.4337.

[9] C. Fronsdal, Phys. Rev 116, 778 (1959); M. Kruskal, Phys. Rev 119, 1743 (1960);

G. Szekers, Publ. Mat. Debreca 7, 285 (1960). 
[10] J. Stachel, "The Hole argument and some physical and philosophical implications" Living. Rev. Relativity 17 (2014) 1.

"Hole Argument" https://en.wikipedia.org/wiki/Hole_argument

[11] T. Padmanabhan, S, Chakraborty, and D. Kothawala, "Spacetime with zero point length is two-dimensional at the Planck scale", Gen. Rel. Grav. 48 (2016) 55.

T. Padmanabhan, "The Atoms Of Space, Gravity and the Cosmological Constant", Int. Jour. Mod. Phys. D 25 (2016) 1630020.

[12] E. Spallucci and A. Smailagic, "Regular Black Holes from semi-classical down to Planck size", arXiv : 1701.04592.

[13] A. Almheiri, D. Marolf, J. Polchinski, and J. Sully, "Black Holes: Complementarity or Firewalls?", arXiv : 1207.3123.

[14] G. ' t Hooft, "The firewall transformation for black holes and some of its implications" arXiv : 1612.08640.

G. 't Hooft, "On the quantum structure of a black hole". Nuclear Physics B 256 (1993) 727.

[15] F. Klinkhamer, "A new type of nonsingular black-hole solution in general relativity" Mod. Phys, Lett. A 29 (2014) 1430018.

S. Bernadotte and F.R. Klinkhamer, Bounds on length scales of classical spacetime foam models, Phys. Rev. D 75, 024028 (2007); arXiv:hep-ph/0610216.

[16] L. Susskind, L. Thorlacius, J. Uglum, The Stretched Horizon and Black Hole Complementarity". Phys. Rev. D48 (1993) 3743; arXiv:hep-th/9306069 [hep-th].

[17] S. Weinberg, "Ultraviolet divergences in quantum theories of gravitation" General Relativity : An Einstein centenary survey, Eds. S. Hawking and W. Israel, Cambridge University Press (1979) p. 790.

M. Reuter and F. Saueressig, "Quantum Einstein Gravity" arXiv: 1202.2274

R. Percacci, "A short introduction to Asymptotic Safety" arXiv: 1110.6389.

D. Litim, "Renormalization group and the Planck scale" arXiv: 1102.4624.

S. Nagy, "Lectures on Renormalization and Asymptotic Safety", arXiv : 1211.4151.

M. Niedermaier, "The Asymptotic Safety Scenario in Quantum Gravity-An Introduction" , gr-qc/0610018.

[18] A. Bonano and Reuter. "Renormalization group improved black hole spacetimes" Phys. Rev. D62 (2000) 043008.

[19] M. Reuter and F. Saueressig, "Asymptotic Safety, Fractals and Cosmology", arXiv: 1205.5431. 
[20] L. Nottale, Scale Relativity And Fractal Space-Time: A New Approach to Unifying Relativity and Quantum Mechanics (World Scientific 2011)

L. Nottale, Fractal Space-Time and Micro-physics (World Scientific 1993).

[21] J.F.Donoghue, Phys. Rev. Lett. 72, 2996, (1994); Phys. Rev. D50, 3874, (1994).

[22] R. Miron, D. Hrimiuc, H. Shimada and S. Sabau, The Geometry of Hamilton and Lagrange Spaces ( Kluwer Academic Publishers, Dordrecht, Boston, 2001 ).

R. Miron, Lagrangian and Hamiltonian geometries. Applications to Analytical Mechanics, arXiv: 1203.4101 [math.DG].

[23] S. Vacaru, "Finsler-Lagrange Geometries and Standard Theories in Physics: New Methods in Einstein and String Gravity" [arXiv : hep-th/0707.1524].

S. Vacaru, P. Stavrinos, E. Gaburov, and D. Gonta, "Clifford and Riemann-Finsler Structures in Geometric Mechanics and Gravity" (Geometry Balkan Press, 693 pages).

[24] G. Amelino-Camelia, L. Freidel, J. Kowalski-Glikman, and L. Smolin, "The principle of relative locality", Phys. Rev. D84 (2011) 084010.

G. Amelino-Camelia, L. Freidel, J. Kowalski-Glikman, and L. Smolin, "Relative locality: A deepening of the relativity principle", Gen. Rel. Grav. 43 (2011) 2547.

[25] L. Barcaroli, L. Brunkhorst, G. Gubitosi, N. Loret and C. Pfeifer, "Hamilton geometry: Phase space geometry from modified dispersion relations" arXiv : 1507.00922.

[26] M. Letizia and S. Liberati, "Deformed relativity symmetries and the local structure of spacetime" arXiv: 1612.03065.

L. Barcaroli, L. Brunkhorst, G. Gubitosi, N. Loret and C. Pfeifer, "Planck-scalemodified dispersion relations in homogeneous and isotropic spacetimes" arXiv : 1612.01390.

I. Lobo, N. Loret and F. Nettel, "Investigation on Finsler geometry as a generalization to curved spacetime of Planck-scale-deformed relativity in the de Sitter case" arXiv : 1611. 04995.

[27] C. Castro, "On Maximal Proper Force, Black Hole Horizons and Matter as Curvature in Momentum Space" submitted to the IJGMMP, Jan. 2017.

C. Castro, "On Dual Phase Space Relativity, the Machian Principle and Modified Newtonian Dynamics" Progress in Physics 1 (April 2005) 20.

[28] C. Castro, "Solutions to the Gravitational Field Equations in Curved Phase-Spaces" to appear in the EJTP, 2017.

[29] V. Kupriyanov and R. Szabo, " $G_{2}$ structures and quantization of non-geometric M-theory backgrounds" arXiv: 1701.02574. 